\section{[gw22-e0207] BALLOON DILATION OF RHEUMATIC MITRAL STENOSIS WITH TRICUSPID REGURGITATION IN PATIENTS WITH RECENT ANALYSIS OF THE MEDIUM-TERM EFFICACY}

Zhangqiang Chen, Lang Hong, Hong Wang, Lixiang Lu, Qiulin Yin, Hua Tai Li Jiangxi Provincial People's Hospital

10.1136/heartjnl-2011-300867.598

Objective To investigate the efficacy and safety of percutaneous balloon mitral valvuloplasty (PBMV) treatment in patients of rheumatic heart disease combined with mitral stenosis and tricuspid valve regurgitation.

Methods 100 patients with mitral stenosis companied by tricuspid valve regurgitation selected in our hospital from January 2000 to January 2011 due to rheumatic heart disease, of which aged $30-65$ years old (average age $46 \pm 11$ years), male 42 cases, female 58 cases, mitral valve area $0.6-1.1 \mathrm{~cm}^{2}$ (an average of $0.9 \pm 0.2 \mathrm{~cm}^{2}$ ), tricuspid regurgitation area $6.0-14 \mathrm{~cm}^{2}$ (average $12.6 \pm 5.5 \mathrm{~cm}^{2}$ ). each expansion increased $0.5-1 \mathrm{ml}$, so that the expansion of the end of the left atrial pressure decreased more than $50 \%$, or left atrial pressure reached an average below 15 $\mathrm{mm} \mathrm{Hg}$ and diastolic mitral valve area rumbling noise disappearing or weakening significantly.

Results The mitral valve areas were $1.6 \pm 0.3 \mathrm{~cm}^{2}$ in 60 patients after percutaneous balloon mitral valvuloplasty, increasing significantly $(p<0.01)$ than the preoperative $0.9 \pm 0.3 \mathrm{~cm}^{2}$; the postoperative tricuspid regurgitation areas were $(1.8-17) \mathrm{cm}^{2}$ (average $6.2 \pm 4.0 \mathrm{~cm}^{2}$ ), was significantly reduced $(\mathrm{p}<0.01)$ than the preoperative $(2.8-26) \mathrm{cm}^{2}$ (average $10.3 \pm 6.4 \mathrm{~cm}^{2}$ ); the postoperative left atrial diameter $(45.4 \pm 7.4) \mathrm{mm}$, significantly reduced $(p<0.01)$ than the preoperative $(49.7 \pm 7.9 \mathrm{~mm})$, and the postoperative right atrial diameter $(28.7 \pm 5.6) \mathrm{mm}$, was significantly reduced $(p<0.01)$ compared to the preoperative values $(46.5 \pm 6.3) \mathrm{mm}$; the postoperative average of left atrium pressure $(15.6 \pm 6.1) \mathrm{mm} \mathrm{Hg}$ significantly reduced $(p<0.01)$ compared with the preoperative $(25.5 \pm 6.6) \mathrm{mm} \mathrm{Hg}$, the postoperative right atrial pressure $(13.2 \pm 2.4) \mathrm{mm} \mathrm{Hg}$ decreased significantly $(p<0.01)$ compared with preoperative $(18.5 \pm 4.7) \mathrm{mm} \mathrm{Hg}$; rumbling -like diastolic murmur from mitral valve weakened from severe to mild degree, Systolic murmur-like heard from mitral valve from the operative $0-1 / 6$ degree up to 1-2/6 degree, The symptom of chest tightness and short of breath was alleviated. All cases followed up for $2-9$ years (average $75 \pm 32$ months), without complications.

Conclusion Percutaneous balloon dilatation for treatment of rheumatic heart disease combined with mitral stenosis and tricuspid valve regurgitation in patients could alleviate the symptoms and reduce size of tricuspid regurgitation, improve the quality of life, with good efficacy and safety. Keywords rheumatic heart disease; mitral stenosis with tricuspid valve regurgitation; percutaneous balloon dilatation; effect 